\title{
Membranes are more mosaic than fluid
}

\author{
Donald M. Engelman'
}

\section{The wealth of new data on membrane protein structures and functions is changing our general view of membrane architecture. Some of the key themes that are emerging are that membranes are patchy, with segregated regions of structure and function, that lipid regions vary in thickness and composition, and that crowding and ectodomains limit exposure of lipid to the adjacent aqueous regions.}

Given their biological importance, membranes have been surprisingly neglected by biochemists until recently. Perhaps this is understandable in view of the technical hurdles that working with them presents. Most methods require purification and observation in aqueous environments alien to the molecular design of a membrane, and so the field had to rely on oversimplified views that still dominate the texts and teaching in this area. But now we have a rising number of high-resolution structures, an abundance of functional data and an evolving conceptual basis for framing more pointed questions. This is leading to a great expansion of interest in the area. Articles in this Insight expose current views of the importance, findings and concepts in membrane biology in some regions of the emerging landscape.

The reductionist view of biology, to which many adhere, rests in part on the structure-function hypothesis: that the structures we find are there for specific functional reasons selected by evolution. In the case of membranes, we might start with the origin of life, noting that compartmentalization is essential for an organism, and that with compartmentalization must come specific ways to surmount the barrier defining the boundary of the compartment - the membrane. Thus, the lipid bilayer, which spontaneously forms permeability barriers surrounding aqueous interiors, must be modified by macromolecules for the uptake of nutrients and the disposal of waste. Further refinements led to the use of the barrier for its energy-storage properties and to the creation of ways to pass information between a cell and its environment.

To frame a context for the reviews that follow, a few general perspectives are presented briefly below. To develop these themes fully would require a much longer text (perhaps a book?), so only representative references are given here, and use is made of the references in the longer treatments by the other authors.

\section{Patchiness in the membrane plane}

An influential step in the study of membranes was taken with the development by Singer and Nicholson in 1972 of the 'fluid mosaic model ${ }^{11}$, which pulled together findings and ideas from the preceding decade. The model has become the standard conceptualization of membrane architecture and is shown redrawn in Fig. 1a as it appears in virtually all biochemistry texts. As important and insightful as this model has been, the emergence of new findings during the passage of 33 years has weakened the generalizations it contains, and it is now appropriate to examine some of them more closely. The model includes the ideas that the proteins of a membrane are dispersed, are at low concentration and that they match the hydrophobic dimension of an unperturbed lipid bilayer with peripheral belts of exposed hydrophobic side chains. The lipid is seen as a sea in which mainly monomeric proteins float unencumbered, and the bilayer surface is exposed directly to the aqueous environment.
Each of these ideas is misleading. Most of the authors of the following reviews write of the preferential associations of molecules in the membrane plane, and as an introduction I suggest that such associations are expected, that membranes are typically crowded and that their bilayers vary considerably in thickness.

Is a membrane a random two-dimensional liquid? In the Singer-Nicholson model, molecules are distributed randomly in two dimensions. But we know from first principles and from experimental observation that non-randomness is the rule. Consider a mixture of $n$
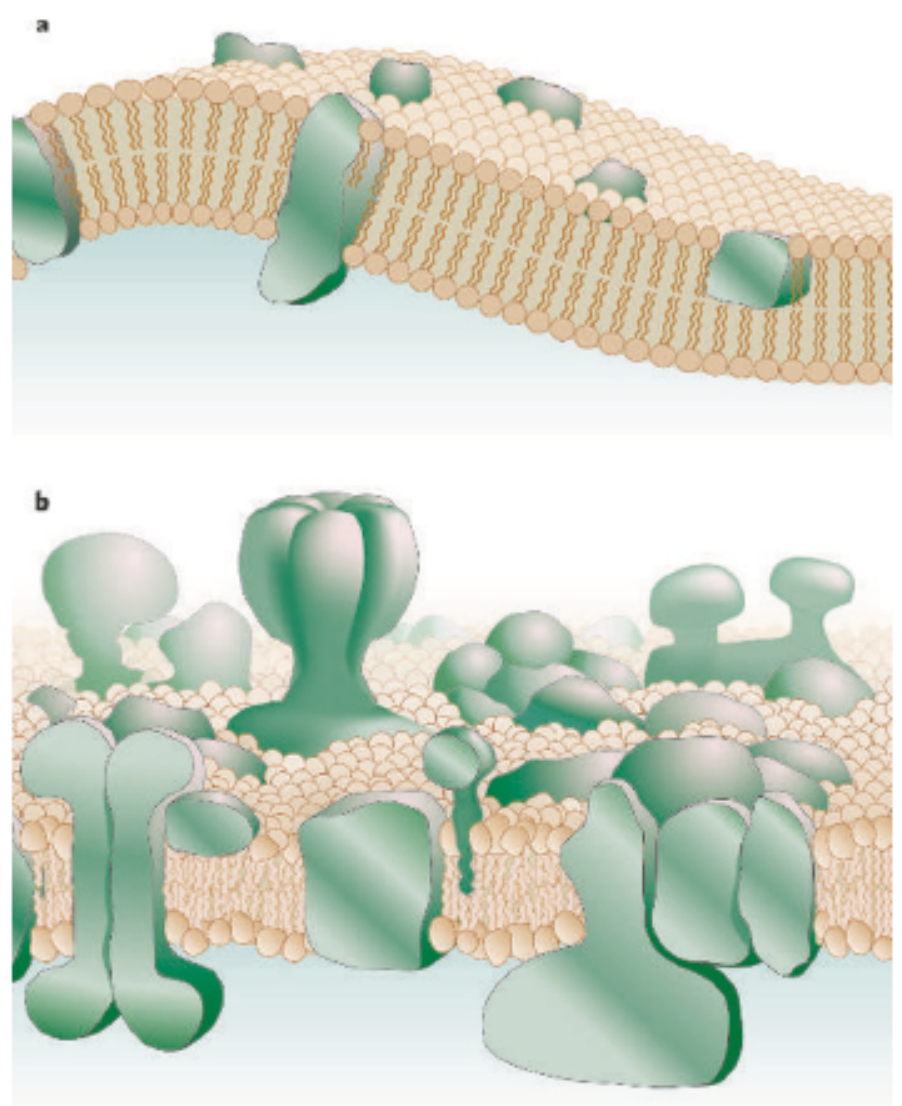

Figure 1 | General models for membrane structure. a, The Singer-Nicholson 'fluid mosaic model' (ref. 1). b, An amended and updated version. 
lipid and protein components in a membrane. The planar distribution can be random only if all pairwise interaction energies of the $n$ different molecular species are within thermal energies (about $0.6 \mathrm{kcal} \mathrm{mol}^{-1}$ at room temperature) of each other. In a plasma membrane there are many species of lipids and proteins. The Escherichia coli genome, for example, codes for more than a thousand putative helical transmembrane proteins ${ }^{2}$, giving more than half a million kinds of pairwise combinations. A narrow range of interaction energies is a highly improbable condition given the range of known intermolecular interactions from hydrogen bonds, packing, electrostatics and the hydrophobic effect. Indeed, simply rotating a pair of identical helices against each other or changing a single interfacial side chain can result in interaction variations of several times $k T$ (refs 3,4$)$. Thus, it should have been expected that regions of biased composition would exist and that the environments of proteins should vary, because it is highly improbable that interaction energies will match each other across all protein and lipid species in a membrane. Time-invariant complexes, transient associations and biased distributions should be the norm. Evolution, ever seeking to exploit the natural tendencies of molecules, has seized the opportunity to craft functional associations, and it is clear that there are functional protein complexes, separated lipid compositional areas and regions of functional specialization, although we do not yet know their extent.

Many experimental observations now support the patchiness of membranes as a principle. A clear case is that proteins are mainly oligomeric. In the thoughtful database compiled by White (http://blanco.biomoluci.edu/Membrane_Proteins_xtal.html), almost all of the unique structures are oligomeric, and many are heterooligomers. Such oligomers are formed using strong associations and are resistant to dissociation by detergent. The excellent folding discussion offered here by Bowie (see page 581) implies that the side-to-side helix associations guiding folding also guide oligomer formation. More associations should be revealed when better means are found to extract them from or observe them in a membrane, and improved methods that reveal larger complexes are appearing ${ }^{5}$. Views of the participation of membrane proteins in organizing large functional complexes are beginning to emerge ${ }^{6}$. In common with proteins, lipids also tend to group together, forming lipid-lipid and lipid-protein interactions. Many lipids are seen in crystal structures to form specific complexes with proteins, most famously in the only structure of an entire membrane that we know - the purple membrane from Halobacterium salinarium ${ }^{7}$. A large body of literature shows that lipids on their own form regions of separated composition in the plane of pure lipid vesicles, as discussed here in the review by Maxfield and Tabas (p.612), who also examine the role of lipids in disease. The ongoing discussion of 'rafts' is a case in point ${ }^{8}$. Further, distortions of the membrane thickness through lipid-protein interactions will create strained regions, as argued below. So, it would seem that patchiness is the order of the day.

Functional patchiness underlies ideas developed in three of the reviews that follow. McLaughlin and Murray (p. 605) discuss the idea of spatial organization imposed by electrostatic interactions as a way to understand the diverse functions of bisphosphophosphatidylinositol $\left(\mathrm{PIP}_{2}\right)$. The discussion of the requirements created by the need for organelle identity, maintenance and function, presented by Behnia and Munro (p. 597), also implies membrane regionalization. It could further be argued that the functional correlates of the membrane curvature discussed by McMahon and Gallop (p. 590) depend on planar segregation of membrane contents and of curvature-inducing proteins when vesicles are formed.

\section{Membrane thickness}

What determines the thickness of a membrane lipid bilayer? The fluid mosaic model posits that "the structures of the lipid in the membrane and of the lipid in isolated aqueous dispersion are closely similar ${ }^{n}$ and that "hydrophobic and hydrophilic interactions are to be maximized and the lowest free energy state is to be attained for the intact membrane in an aqueous environment ${ }^{n 1}$. It follows that membrane proteins
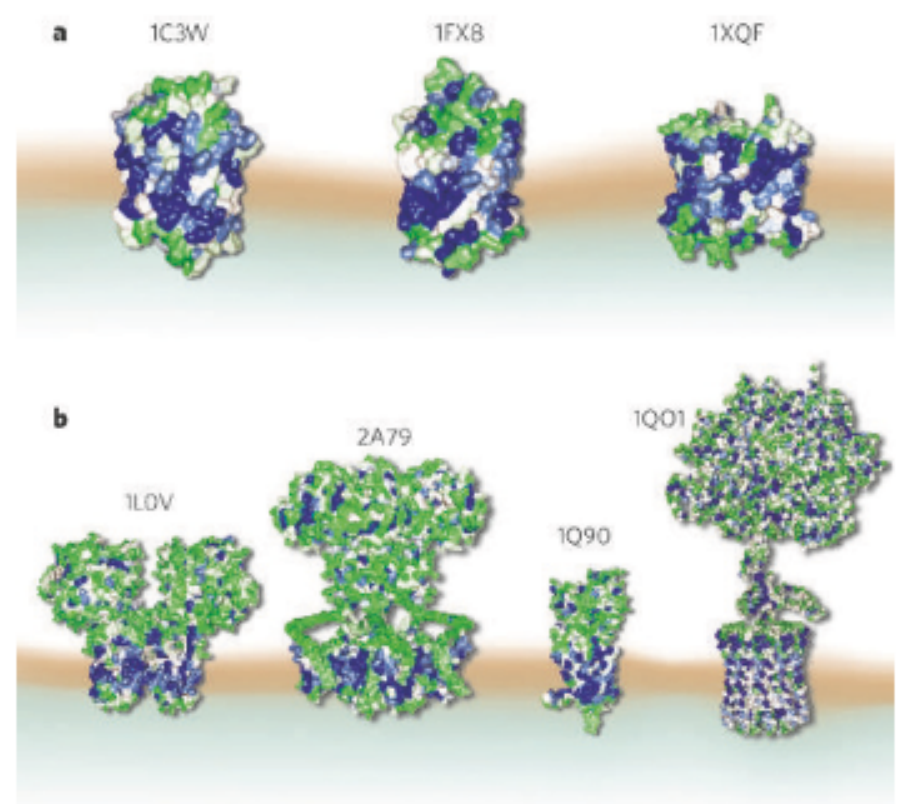

c

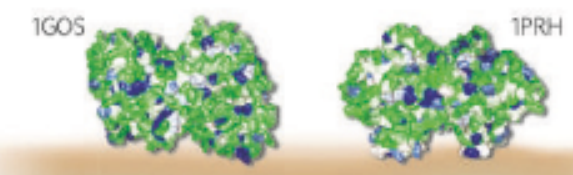

Figure 2 | Known structures for membrane proteins ${ }^{4{ }^{14-2}}$ represented using the energy scale of ref. 33. a, Proteins largely within the membrane bilayer. b, Proteins with large extramembrane regions. c, Proteins covering large regions of lipid $^{34}$. The green indicates amino acids with a favourable interaction with the hydrophobic lipid region, blue a favourable interaction with water. Calculations are by Jonathan Sachs and Michael Strickler.

should have evolved their hydrophobic regions to fit the lipid dimension perpendicular to the membrane plane, since the exposure of hydrophobic surface area to water is unfavourable by about $25 \mathrm{cal} \AA^{-2}$, and the exposure of, say, about $5 \AA$ of bacteriorhodopsin surface out of the membrane would be unfavourable by about $25 \mathrm{kcal} \mathrm{mol}^{-1}$. Inspection of the known structures shows that they vary in hydrophobic dimension around their peripheries and also from one to another. Something must give - either the protein distorts to match the bilayer or the lipid distorts to match the protein, or both. The fluidity of the lipid and the relative rigidity of the proteins ${ }^{9}$ suggest that it is the main lipid that distorts to match the protein, and both modelling and experiment support this view ${ }^{10,11}$, although protein distortion may occur in extreme cases of protein-lipid mismatch ${ }^{12}$.

If the lipid distorts to cover the hydrophobic area of a membrane protein, the transmembrane dimension of a bilayer in membranes with high protein-to-lipid ratios must be variable. Further, if the distortion is asymmetrical across a bilayer, curvature can result (discussed in the review by McMahon and Gallop, p. 590). Local distortion of the bilayer is likely to influence protein interactions, for example, the peripheral energy of distorting the bilayer may enhance interactions that reduce the peripheral contour length. What effect the energy of distorting the bilayer might have on the protein itself is not known, but might have functional relevance for cases where the protein varies its transmembrane conformation in the course of activity.

\section{Area occupancy by protein and lipid}

How much of the membrane bilayer area is occupied by protein? In general, we do not know the answer to this question, yet the answer 
strongly influences concepts of membrane organization. Further, proteins may occupy small areas at the bilayer level but have large ectodomains covering lipid and creating steric restrictions. Most drawings of the fluid mosaic model greatly exaggerate the lipid area in both senses - the area occupied by protein and the area covered by protein are shown as small and zero, respectively.

Membrane protein shapes vary greatly, as shown in Fig. 2. Some are largely contained within the bilayer ${ }^{414-18}$, as in the examples in Fig. 2a. Many protein complexes, such as the ATP synthase, have large structures outside the lipidic region that will create steric contacts and other interactions outside the bilayer ${ }^{19-26}$ (Fig. 2b). These may occupy larger areas in projection onto the membrane than do the transmembrane regions. For example, the F1 ATPase ectodomains occupy about four times the membrane surface area of the transmembrane region ${ }^{23}$. Proteins anchored by single helices or by lipidic anchors such as fatty acids can cover large regions of a membrane with protein surface ${ }^{2728}$, as in the examples shown in Fig. 2c. Interactions of the ectodomain structures are known to be functionally important in many cases, such as the tyrosine kinase receptors ${ }^{29}$. Inspection of the examples in Fig. 2 shows that the exposure of membrane lipid surface may be rather small, for example when a plasma membrane is viewed from either the cytoplasm or the extracellular milieu. However, some proteins associate and dissociate with lipid as part of their function ${ }^{30}$, so some lipid exposure must be maintained. Whether lipid exposure to the cytoplasm might be used to control or focus such interactions is at present unexplored.

\section{Fluidity in the context of order}

Although most membranes exhibit fluidity, with rapid diffusion observed for some lipid and protein species in the plane of a membrane, recent measurements using single-particle tracking reveal a complex set of restrictions on protein lateral mobility. These include directed motion, confined motion and anomalous diffusion ${ }^{31}$. Although the observation that some proteins can move relatively freely suggests that a subset may not be in larger assemblies, crowding, ectodomain collisions, transbilayer interactions, adhesion sites and cytoskeletal structure produce a variety of restrictions on the motion of most proteins and lipids ${ }^{32}$. Not yet considered are the additional constraints imposed by lipid-protein interactions through complex formation and thickness perturbation.

Fluidity must be reconciled with order. It follows that the patchiness of many membranes must be local enough for there to be channels of lipid separating regions of protein assemblies, but this constraint would still allow large segregated regions. The sizes and variability of segregated regions are still to be established.

\section{Consequences for current concepts}

The concepts developed above lead us to a view of a membrane that has variable patchiness, variable thickness and higher protein occupancy than has generally been considered. It will be a challenge to the immense and excellent body of work on pure lipid systems to assimilate the perturbations by proteins. Of course, there will be variability - myelin membranes are low in protein content; photosynthetic membranes high. But, while we await improved measurements, the modified view sketched in Fig. $1 \mathrm{~b}$ is suggested as a guide to thinking, and as a context for the functional interactions that are discussed in the reviews that follow.

1. Singer, S L\& Nicolson, G.L.The fluid mosaic model of the structure of cell membranes. Sdence 175, 720-731(1972).

2. Liu Y, Engelman, D. M. \& Gerstein M. Genomic analysis of membrane protein families: abundance and conserved motits. Genome Bial. 3, 0054.1-0054.12 (2002).

3. Doura, A. K, Kobus, F. L,Dubrowsky, L_Hibbard E \& Fleming K G. Sequence context modulates the stability of a GxoxG-mediated transmembrane hellix-helix dimer. L. Mal. Bial. 341, 991-998 (2004)

4. Adams, P.D. Engelman, D. M. \& Brunger, A. T. Improved prediction for the structure of the dimeric transmembrane domain of glycophorin A obtained through global searching. Proteins 26, 257-261 (1996).

5 Stenberg, F.etal. Protein complexes of the Escherichia colicell envelope $\mathcal{L}$. Bial. Chem. 280 34409-34419 (2005).

6. Wong W, Scott, ID. AKAP signalling complexes: focal points in space and time Nature Rev. Mal. Cell Bial. 5, 959-970 (2004)

7. Grigorieff, N, Ceska, T.A, Downing, K.H, Baldwin, L M. \& Henderson, R. Electroncrystallographicrefinement of the structure of bacteriorhodopsin. I.Mal. Bial. 259, 393-421 (1996)

8. Brown, D.A.\& London, E. Functions of lipidrafts in biologicalmembranes. Annu. Rex Cell Dev. Bial. 14, 111-136 (1998).

9. Zaccai, G. How soft is a protein? A protein dynamics force constant measured by neutron scattering.Sdence 288, 1604-1607(2000).

10. Petrache, H.I.et al.Hydrophobic matching mechanism invest gated by molecular dynamics simulations. Langmuir 18 , 1340-1351 (2002)

11. Mitra, K, Ubarretxena-Belandia, L, Taguchi, T, Warren, G, \& Engelman, D.M. Modulation of the bilaver thic kness of exocytic pathway membranes by membane proteins rather than cholesterol. Poc Natl Acad. Sci. USA 101, 4083-4088(2004).

12. Williamson, LM, Alvis, S.J_East,J.M. \& Lee, A. G Interactions of phospholipids with the potassium channel KcsA. Biaphys J.83, 2026-20B8 (2002).

13. Peroza, E, Kloda, A, Cortes, D. M.\& Martina, B. Physicalprinciplesunderlying the transduction of bilayer deformation forces during mechanosensitive channel gating Nature Struct Biol. 9, 636-637(2002)

14. Abramson, 1 et al.Structure and mechanism of the lactose permease of Escherichia cali. Sdence 301, 610-615 (2003).

15. Fu, D.etal.Structure of a glycerol-conducting channel and the basis for its selectivity. Sdence $290,481-486(2000)$

16. Luecke, H, Schobert, B, Richter, H. T, Cartaillex, I. P. \& Lanyi, I K Structure of bacteriorhodopsinat 155 Aresolution. L. Mal. Bial. 291, 899-911 (1999).

17. Zheng $L_{,}$Kostrewa, D, Berneche, S. Winkler, F.K.\& L X, X. The mechanism of ammonia transport based on the crystal structure of AmtB of Escherichia call. Proc. Natl Acid Sci. USA $101,17090-17095$ (2004).

18. A bramson, 1 et al. The structure of the ubiquinol oxidase from Escherichiacall and its ubiquinone binding site Nature Struct. Bial. 7,910-917 (2000).

19. Iverson, T.M, Luna-Chavez, C, Cecchini, G. \& Rees, D. C. Structure of the Escherichia coll $^{2}$ fumarate reductase respiatory complex. Science 284, 1961-1966 (1999)

20.Long S. B. Campbell, E B.\& Mackinnon, R.Cystal structure of a mammal lan voltagedependent Shaker family $\mathrm{K}^{*}$ channel.Science 309,897-903(2005).

21. Kur isu G, Zhang $H$, Smith, 1 L \& Cramer, W. A. Structure of the cytochrome b6 f complex of oxygenic photosynthesis: tuning the cavity. Science 302, 1009-1014 (2003).

22. Miyazawe, A, Fuilyoshi, Y.\& Unwin, N (200B) Structure and gating mechanism of the acetylcholine receptor pore. Nature 423,949-955.

23. Stock, D, Leslie, A.G.\& Walkec, L.E.Molecular architecture of the rotary motor in ATP synthase.Sdence 286, 1700-1705 (1999).

24. Stroebel, D, Choquet, Y, Popot, IL \& P cot, D.An atypical haem in the cytochrome b(6)f complex Nature 426, 413-418 (2003).

25. Xia, D. et al. Crystal structure of the cytochrome bc 1complex from bovine heart mitochondria Science Z7, 60-66 (1997).

26. Zouni.A.etal. Crystal structure of photosystem II from Synechococcus elongatus at $38 \AA$ resolution. Nature 409, 739-743 (2001).

77. Binda, C, Newton-Vinson, P, Hubalek, F, Edmondson, D. E.\& Mattevi, A. Structure of human monoamine oxidase $\mathrm{B}$, a drug target for the trea tment of neurologicaldisorders. Nature Struct. Biol. 9, 22-26 (2002)

28. Bracey, M.H_. Hanson, M.A.Masuda, K.R. Stevens, R. C. \& Cravatt, B.F.Structural adaptations in a membrane enzyme that terminates endocannabinoid signaling. Sdence 298,1793-1796 (2002).

29. Ferguson $\mathrm{K}$ M. et a. EGF activates its receptor by removing interactions that autoinhibit ectodomain dimerization. Mal. Cell 11,507-517(2003)

30.Newton, A.C. Regulation of the $A B C$ kinases byphosphorylation. protein kinase $C$ as a paradigm. Blochem. J.370, 361-371 (2003)

31. Kusumi,A. et al.Paradigm shift of the plasma membane concept from the two-dimensional continum fluid to the partitioned fluid: high-speed single-molecule tracking of membrane molecules. Amu Rev. Biophys Blomal. Struct. 34, 351-378(2005).

32. Saxton, M.J.\& lacobson, K. Single-particle tracking:applic ations to membrane dynamics. Annu Rev. Biophys. Biomal. Struct. 26, 373-399 (1997).

33. Hessa. T. et al. Recognition of transmembrane helices by the endoplasmic reticulum translocon. Nature 433, 377-381(2005).

34.Picot, D. Loll, P. I \& Garavita, R. M. The X-ray structure of the membrane protein prostaglandin H2 synthase-1. Nature 367, 243-249 (1994).

Acknowledgements I thank the members of my laboratory for discussions, and the US National Institutes of Health and NFCR for support.

Author Information Reprints and permissions information is available at npg_nature.com/reprintsandpermissions. The authors declare no competing financial interests. Correspondence should be addressed to D.M.E.

(don@mail.csbyale.edu). 